\section{Selinexor combined with cladribine, cytarabine, and filgrastim in relapsed or refractory acute myeloid leukemia}

Inactivation of tumor suppressor proteins (TSP) and growth regulatory proteins (GRP) is a critical mechanism contributing to tumorigenesis. As many TSP and GRP require nuclear localization for their activities, excess nuclear export can lead to their functional inactivation in malignant cells. Selinexor (KPT-330) is an oral, first in class, selective inhibitor of nuclear export (SINE) compound that blocks Exportin 1 (XPO1). Blockade of XPO1 causes TSP to accumulate in the nucleus of all cells, restoring tumor suppressing pathways that lead to selective elimination of genetically damaged cells ${ }^{1-4}$

In this paper, we report our preclinical and clinical experience using selinexor in combination with chemotherapy for the treatment of relapsed or refractory acute myeloid leukemia (rrAML).

We first tested the therapeutic effect of selinexor in a genetically-defined murine acute myeloid leukemia (AML) model. ${ }^{5}$ The combination of selinexor and AraC prolonged the overall survival of tumor-bearing mice compared to chemotherapy alone $(P<0.001$, Figure $1 \mathrm{~A})$. The selinexor plus AraC combination group had better tumor response as shown by the decreased white blood cell counts when compared to the chemotherapy alone group (Figure 1B).

We next assessed the effect of selinexor and chemotherapy on normal murine hematopoiesis. Treatment with selinexor plus AraC decreased the frequency of LSK (lineage ${ }^{-}, \mathrm{Sca}-1^{+}, \mathrm{KIT}^{+}$) hematopoietic stem and progenitor cells in the bone marrow of mice (Figure 2A), while common lymphoid progenitors (CLP, $\mathrm{Lin}^{-} \mathrm{Il} 7 \mathrm{ra}^{+} \mathrm{c}-\mathrm{Kit}^{+} \mathrm{Sca}^{-} 1^{+}$) were significantly lower in both the selinexor alone and selinexor plus AraC combination groups compared to the vehicle or AraC only groups (Figure 2B). Transplantation of whole bone marrow from selinexor plus AraC mice into congenic recipients was associated with decreased donor engraftment with a median of $18 \%$ donor cells (Figure 2C-D). The decrease in engraftment potential for the selinexor plus Ara-C treated mice was magnified in a secondary transplantation experiment with as low as $<5 \%$ donor cell engraftment. Furthermore, at six months post-transplant, LSK and CLP populations were significantly lower in secondary transplanted mice treated with the combination of selinexor plus AraC (Figure 2G-H). In summary, our preclinical data demonstrates increased antitumor effects with the combination of selinexor and cytarabine but with the potential for hematopoietic toxicity.

Based on our preclinical data, we performed an openlabel phase $1 / 2$ study of selinexor in combination with cladribine, cytarabine and G-CSF (CLAG) for the treatment of patients with rrAML. Eligible patients were 1870 years of age with a diagnosis of rrAML and one of the following: 1) primary refractory disease following $\leq 2$ cycles of induction chemotherapy, 2) first relapse with no prior unsuccessful salvage chemotherapy, or 3) relapsed or refractory to treatment with a DNA hypomethylating agent. Patients were required to have an Eastern Cooperative Oncology Group (ECOG) performance status $\leq 2$ and adequate organ function defined as: 1) creatinine clearance $\geq 50 \mathrm{~mL} / \mathrm{min}, 2$ ) aspartate aminotransferase, alanine aminotransferase, and total bilirubin $\leq 2$ times the institutional upper limit of normal and 3) left ventricular ejection fraction of $\geq 40 \%$.
Table 1. Patient characteristics $(\mathrm{N}=40)$.

\begin{tabular}{lc}
\hline Age in Study (median, range), years & $55.5(21-70)$ \\
Female Sex N (\%) & $15(37.5 \%)$ \\
Ethnic Origin N (\%) & \\
Caucasian, Non-Hispanic & $38(95 \%)$ \\
African American, Non-Hispanic & $2(5 \%)$ \\
\hline RFI median, (range), mo & $6.5(0-79)$ \\
Status at Study Entry N (\%) & \\
Primary refractory disease following & $12(30 \%)$ \\
two or less cycles of induction chemotherapy & \\
First relapse with no prior unsuccessful & $27(67.5 \%)$ \\
salvage chemotherapy & \\
Relapsed/refractory to hypomethylating agent & $1(2.5 \%)$ \\
\hline Cytogenetic Risk Classification N (\%) & \\
Favorable & $2(5 \%)$ \\
Intermediate & $27(67.5 \%)$ \\
Poor & $9(22.5 \%)$ \\
Not Available & $2(5 \%)$ \\
\hline
\end{tabular}

RFI: Relapse-free interval, mo: months.

Patients received selinexor $60 \mathrm{mg}$ orally once per day on days $1,5,10$, and 12 , cladribine $5 \mathrm{mg} / \mathrm{m}^{2} /$ day 4 on days $4-8, \mathrm{G}-\mathrm{CSF} 300 \mathrm{mcg}$ SC once per day on days 3-8, and cytarabine $2,000 \mathrm{mg} / \mathrm{m}^{2} /$ day 4 on days $4-8$. No dose modifications were made for selinexor or CLAG chemotherapy. To minimize nausea, all patients received 5 -HT3 antagonists (ondansetron $8 \mathrm{mg}$ or equivalent) starting before the first dose of selinexor and continued two to three times a day as needed.

We enrolled 40 patients between June 6, 2015 and January 28, 2018 (Table 1). Eighteen of 40 patients (45\%) achieved complete remission (CR) or complete remission with incomplete hematologic recovery (CRi), compared with our institutional historical rate of $33 \% \quad(P=0.16)$, therefore, the phase 2 portion failed to meet its primary endpoint. The median duration of remission was 9.1 months. The median event-free and overall survival was 6.1 months $(95 \%$ Confidence Interval [CI]: 4.5-7.8 months) and 7.8 months (95\% CI: 5.7-14.1 months) respectively, and 15 patients $(37.5 \%)$ were alive at the time of the last follow-up. Neutrophil and platelet engraftment occurred at a median time of 28 days (range: 24-58) and 38 days (range: 29-61) respectively. Twentyfour of 40 patients $(60.0 \%)$ proceeded to allogeneic hematopoietic cell transplantation (alloHCT) after treatment on study.

The 30 and 60 day mortality was $2.5 \%(n=1)$ and $7.5 \%$ $(n=3)$ respectively. Gastrointestinal toxicity was manageable with grade III nausea and grade II diarrhea each seen in three $(7.5 \%)$ patients. Grade I-II nausea (23 patients, $57.5 \%$ ), vomiting (23 patients, $57.5 \%$ ), and diarrhea (16 patients, $40 \%$ ) were more common, but in no cases resulted in dose delays or reductions. Three patients were removed from protocol therapy for treatment emergent adverse events (TEAE) - grade III pancreatitis deemed probably related to the study drug, grade IV colitis deemed possibly related to the study drug, and grade IV sepsis deemed unrelated to the study drug.

Relapsed or refractory AML remains a challenging disease entity, with poor response rates and overall survival. ${ }^{6,7}$ Selinexor has modest single-agent efficacy in AML with a CR/CRi rate of $14 \% .^{8}$ With non-overlapping toxicities, combining CLAG and selinexor is a rational 
approach to improving on CLAG, an established regimen in the rrAML setting. In this study, $45 \%$ of treated patients achieved a CR or CRi. The response rate was encouraging for a nonanthracycline-containing salvage regimen, but unfortunately did not differ significantly from our prespecified historical rate of $33 \% \quad(P=0.16)$. These results are comparable to another recent study using selinexor in combination with an anthracyclinecontaining intensive induction regimen, in which the CR/CRi rate was $38 \%(n=8)$ for patients with rrAML. ${ }^{9}$ Sixty percent of patients proceeded to alloHCT, which is similar to the rate of transplant in a large retrospective analysis where 385 of 788 patients $(49 \%)$ went to alloHCT after intensive salvage chemotherapy. ${ }^{10}$

In previous clinical studies of selinexor, the most common TEAE events included nausea, fatigue, anorexia and vomiting that occurs in a dose-dependent manner. ${ }^{8,11}$ Notably, from preclinical studies, anorexia and weight loss with selinexor appears to be centrally mediated and distinct from anorexia and nausea typically associated with chemotherapy. In this study, rates and severity of these toxicities were low for several reasons. First, the flat dose of $60 \mathrm{mg}$ twice per week was chosen as this showed promising efficacy with acceptable toxicity. ${ }^{11}$ Second, aggressive supportive care measures with universal premedication with 5-HT3 antagonists and dexamethasone were initiated prior to beginning selinexor therapy. Only $7.5 \%$ of patients ( 3 of 40 ) were taken off therapy due to adverse effects. The low rates of gastrointestinal grade III-IV TEAE nausea $(3 \%)$ and diarrhea $(3 \%)$, were encouraging and are consistent with rates in selinexor single agent clinical trials.

Hematopoietic recovery was an important safety outcome given the observed hematopoietic toxicity in our preclinical model. Additionally, a recent clinical trial combining selinexor with intensive induction therapy in AML

A

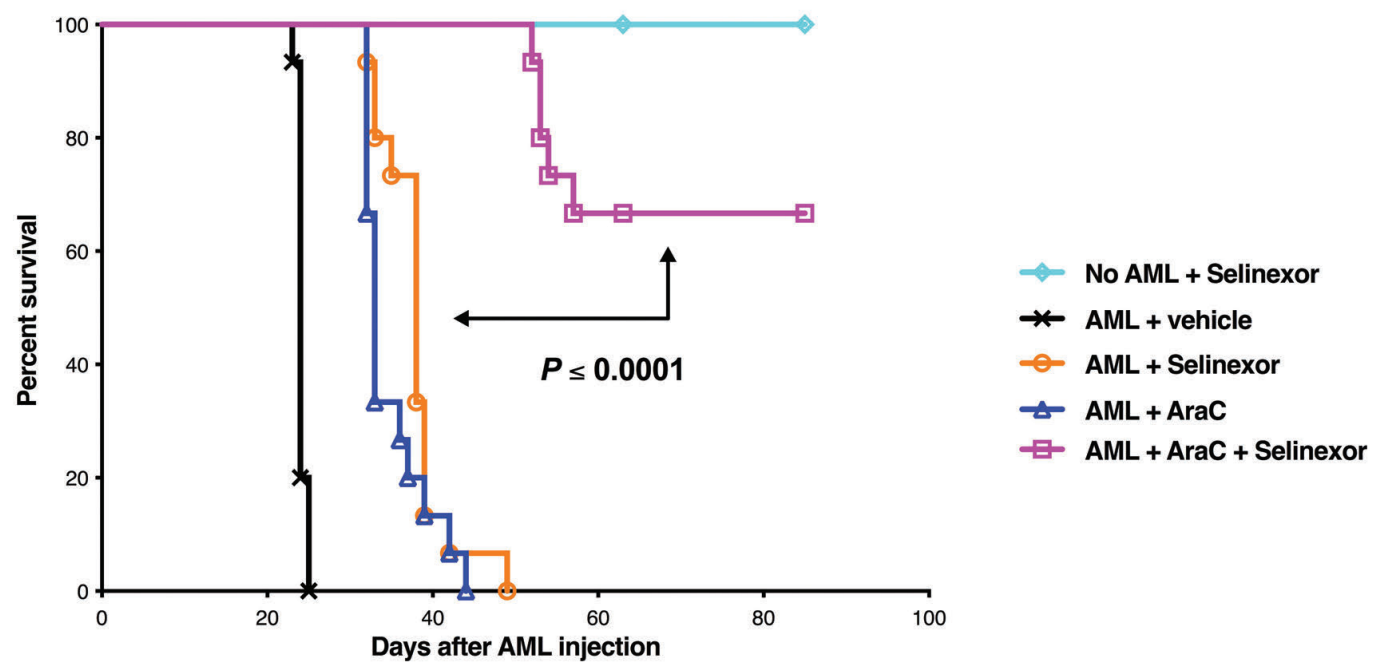

B

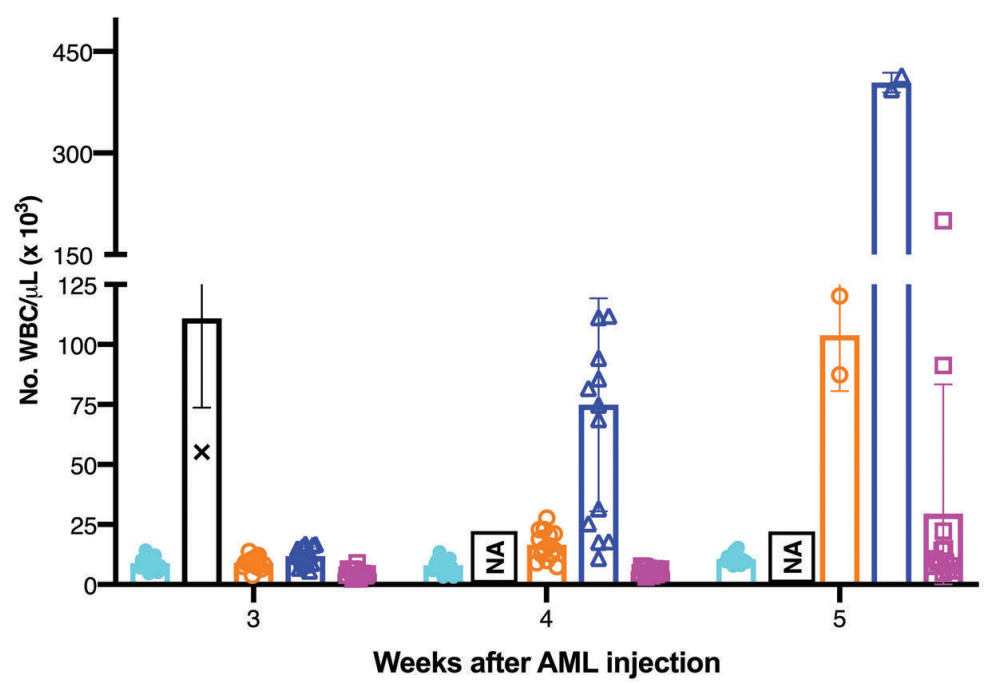

$\begin{array}{ll} & \text { No AML + Selinexor } \\ \times & \text { AML + Vehicle } \\ & \text { AML + Selinexor } \\ \triangle & \text { AML + AraC } \\ \square & \text { AML + AraC + Selinexor }\end{array}$

Figure 1. Selinexor plus AraC combination therapy provides significant survival advantage. Male C57BL/6J mice were injected with primary murine acute myeloid leukemia (AML) tumor $\left(1 \times 10^{6} \mathrm{cells} /\right.$ mouse, intravenously). After three days of engraftment, groups of mice ( $\left.\mathrm{n}=15 \mathrm{each}\right)$ were treated with either vehicle (oral gavage), selinexor only (15 mg/kg; oral gavage), AraC only (200 mg/kg; subcutaneous injection) or the combination of selinexor plus AraC. The drugs were given three days a week for two weeks. (A) Log rank test for comparison of Kaplan-Meier survival curves indicated a significant increase in the survival of the mice treated with selinexor plus AraC combination ( $P \leq 0.0001)$ compared to selinexor or AraC only. (B) The white blood cell counts measured at week 3,4 and 5 after AML injections show a better tumor response in the selinexor plus AraC combination group. Data are pooled from two separate experiments. 
A

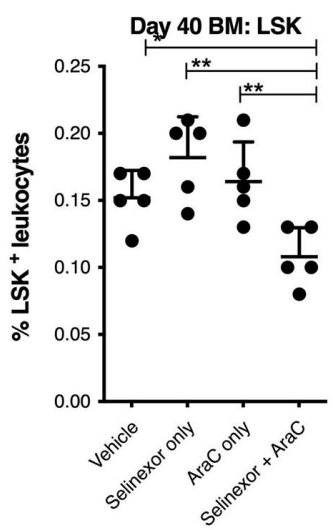

C

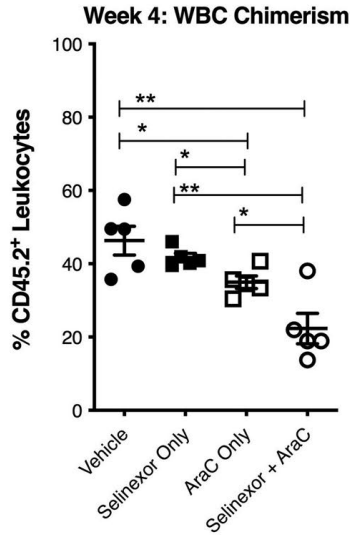

E

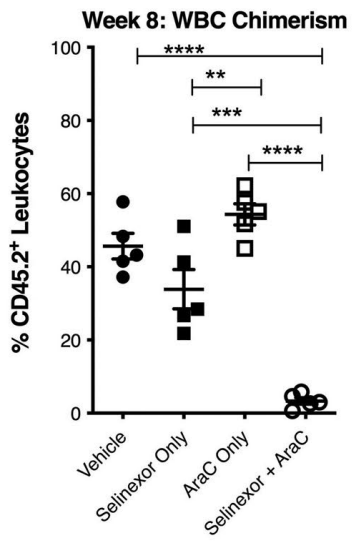

G

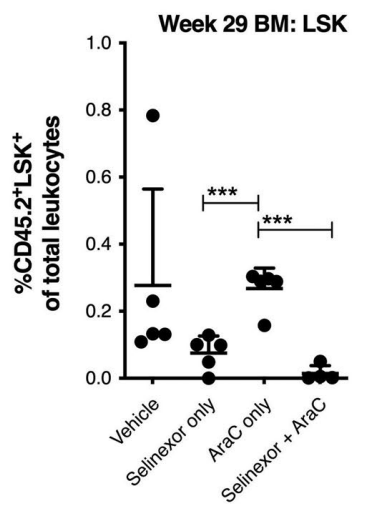

Treatment

B

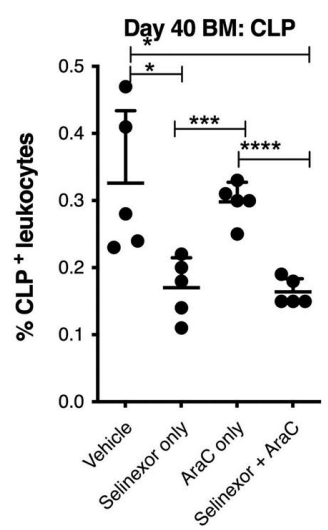

\section{$1^{\circ}$ Transplant}

D

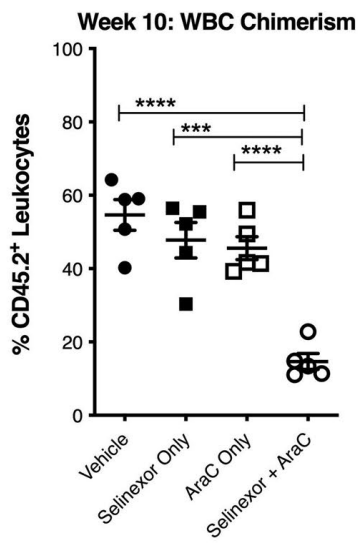

\section{$2^{\circ}$ Transplant}

F

Week 15: WBC Chimerism

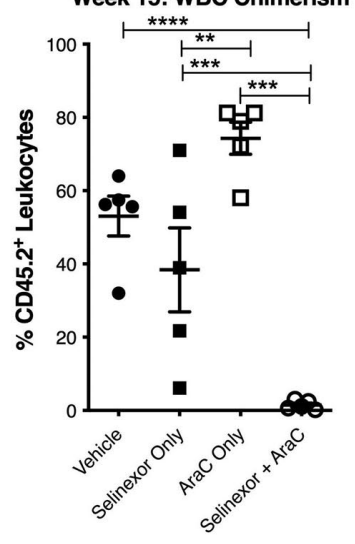

H

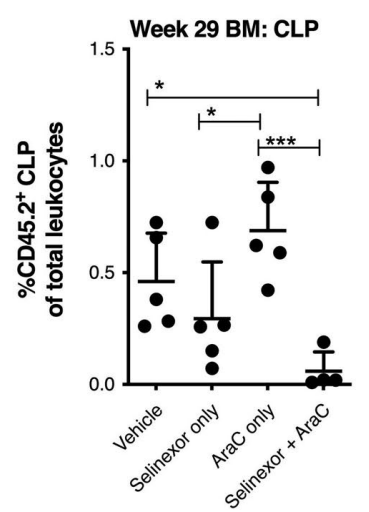

Figure 2. Selinexor plus AraC combination therapy impairs hematopoietic recovery and reduces hematopoietic stem cell engraftment potential in vivo. Treatment: thirty male C57BL/6 J were treated with either vehicle (oral gavage), selinexor only (15 $\mathrm{mg} / \mathrm{kg}$; oral gavage), AraC only (200 mg/kg; subcutaneous injection) or the combination of selinexor plus AraC. There were five mice per group. The drugs were given three days a week for two weeks. (A) The frequency of $\mathrm{Lin}^{-} \mathrm{Sca}^{+} \mathrm{CKit}^{+}$(LSK) cells and (B) $\mathrm{Lin}^{-} \mathrm{Sca}^{+} \mathrm{cKit}^{+} \mathrm{CD} 34^{-}$common lymphoid progenitor cells (CLP) were determined by fluorescenceactivated cell sorting on day 40 post-treatment bone marrow. The CLP subset was significantly reduced in both the selinexor only $(P=0.007)$ and selinexor plus AraC groups $(P<0.0001)$ compared with the AraC only group. Competitive repopulation experiment (C) At four weeks peripheral blood showed reduced engraftment of $\mathrm{CD} 45.2^{+}$cells in the selinexor plus AraC group compared to the selinexor only $(P=0.002)$ and AraC only $(P=0.022)$ groups. (D) At 10 weeks CD45.2 $2^{+}$cells were more significantly reduced in the selinexor plus AraC group compared to the selinexor only $(P=0.0002)$ and AraC only $(P<0.0001)$ groups. Secondary transplant $(\mathrm{E})$ The engraftment efficiency of CD45.2+ donor cells at eight weeks was significantly reduced in the selinexor plus AraC group compared to the selinexor only $(P=0.0005)$ and AraC only $(P<0.0001)$ group. (F) At 15 weeks this effect was more evident between the selinexor plus AraC and the AraC only groups $(P<0.0001)$. Bone evaluation six months post transplantation showed significantly reduced frequency of CD45.2+ LSK (G) and CLP cells $(H)$ in the group treated with the selinexor plus AraC combination compared to $\mathrm{AraC}$ alone with $P=0.001$ and $P=0.001$ respectively. 
using selinexor at a higher dose of $80 \mathrm{mg}$ in combination with high dose cytarabine and mitoxantrone demonstrated an acceptable safety profile but noted prolonged thrombocytopenia and delayed neutrophil recovery. Selinexor may be uniquely disruptive to early megakaryopoiesis through inhibition of thrombopoietin signaling. ${ }^{12}$ This effect occurs during megakaryocytic differentiation from hematopoietic stem cells, and the drug may be minimally toxic to mature megakaryocytes. After intensive chemotherapy, when bone marrow repopulation is occurring, selinexor may induce prolonged or persistent thrombocytopenia. In this clinical trial, the median number of days to neutrophil and platelet recovery were 28 (ANC $\geq 1,000 / \mathrm{mm}^{3}$ ) and 37 days (platelets $\geq 100,000 \mathrm{~mm}^{3}$ in the absence of transfusion) respectively. However, seven patients, who had no morphologic evidence of disease based on their day 30 bone marrow, failed to recover the platelet count prior to proceeding to conditioning chemotherapy and alloHCT. Despite thrombocytopenia, the only grade $\geq$ III bleeding event was grade III hematuria occurring in four $(10 \%)$ patients. Notably, preclinical evidence generated from our group, suggesting that selinexor may adversely affect normal hematopoietic stem cell activity, was not borne out in the clinical trial, as bone marrow aplasia or prolonged neutropenia was not observed. This trial used a lower dose of selinexor and an anthracycline-free regimen, compared with the aforementioned trial of first line AML therapy in which prolonged cytopenias were observed. These data suggest that effects on normal hematopoiesis may be related to the dose and schedule of selinexor as well as the concurrent use of anthracyclines.

In conclusion, data from this study show that CLAG and selinexor can be combined safely, in an anthracycline-free regimen, and provide a bridge to transplant in patients with rrAML. Similar to other studies of selinexor, GI toxicities and thrombocytopenia were notable adverse events. Currently oral second-generation SINE compounds such as eltanexor (KPT-8602) are in clinical development. In preclinical models, the orally bioavailable agent appears to have less central nervous system penetration and potentially less central nervous system mediated effects of anorexia and weight loss potentially allowing more frequent and higher exposure than is possible with selinexor. ${ }^{13}$

Ramzi Abboud, ${ }^{1 *}$ Ezhilarasi Chendamarai, ${ }^{1 *}$ Michael P. Rettig, ${ }^{1}$ Kathryn M. Trinkaus, ${ }^{2}$ Peter A. Riedell, ${ }^{\circ}$ Camille N. Abboud, ${ }^{1}$ Armin Ghobadi, ${ }^{1}$ Iskra Pusic, ${ }^{1}$ Keith Stockerl-Goldstein, Mark A. Schroeder, ${ }^{1}$ Ravi Vij, ${ }^{1}$ Peter Westervelt, ${ }^{1}$ John F. DiPersio and Geoffrey L. Uy'

${ }^{*} R A$ and EC contributed equally as co-first authors.

${ }^{1}$ Division of Oncology, Washington University School of Medicine and 'Biostatistics Shared Resource Siteman Cancer Center, Washington University School of Medicine, St. Louis, MO, USA
${ }^{\circ}$ Current Address: Section of Hematology/Oncology, University of Chicago, Chicago, IL, USA

Funding: The Alvin J. Siteman Cancer Center is supported in part by National Cancer Institute Cancer Center Support grant P30

CA91842. RA is supported by the American Society of Hematology

Clinical Research Training Institute and the National Cancer Institute of the National Institutes of Health under Award Number R25

CA190190.

Correspondence: GEOFFREYL.UY-guy@wustl.edu

doi:10.3324/haematol.2019.236810

Information on authorship, contributions, and financial 2 other disclosures was provided by the authors and is available with the online version of this article at www. haematologica.org.

\section{References}

1. Kim J, McMillan E, Kim HS, et al. XPO1-dependent nuclear export is a druggable vulnerability in KRAS-mutant lung cancer. Nature. 2016; 538(7623):114-117.

2. T. K, M. C, S. F, et al. Selective inhibitors of nuclear export (SINE) block the expression of DNA damage repair proteins and sensitize cancer cells to DNA damage therapeutic agents. Eur J Cancer. 2014; 50(1):83.

3. Etchin J, Montero J, Berezovskaya A, et al. Activity of a selective inhibitor of nuclear export, selinexor (KPT-330), against AML-initiating cells engrafted into immunosuppressed NSG mice. Leukemia. 2016;30(1):190-199

4. Ranganathan P, Kashyap T, Yu X, et al. XPO1 inhibition using selinexor synergizes with chemotherapy in acute myeloid leukemia by targeting DNA repair and restoring topoisomerase II $\alpha$ to the nucleus. Clin Cancer Res. 2016;22(24):6142-6152.

5. Westervelt P, Lane AA, Pollock JL, et al. High-penetrance mouse model of acute promyelocytic leukemia with very low levels of PML-RAR $\alpha$ expression. Blood. 2003;102(5):1857-1865.

6. Breems DA, Van Putten WLJ, Huijgens PC, et al. Prognostic index for adult patients with acute myeloid leukemia in first relapse. J Clin Oncol. 2005;23(9):1969-1978.

7. Chevallier P, Labopin M, Turlure P, et al. A new Leukemia Prognostic Scoring System for refractory/relapsed adult acute myelogeneous leukaemia patients: A GOELAMS study. Leukemia. 2011;25(6):939944.

8. Garzon R, Savona M, Baz R, et al. A phase 1 clinical trial of singleagent selinexor in acute myeloid leukemia. Blood. 2017; 129(24):3165-3174.

9. Wang AY, Weiner $\mathrm{H}$, Green $\mathrm{M}$, et al. A phase I study of selinexor in combination with high-dose cytarabine and mitoxantrone for remission induction in patients with acute myeloid leukemia. J Hematol Oncol 2018;11(1):1-10.

10. Wattad M, Weber D, Döhner K, et al. Impact of salvage regimens on response and overall survival in acute myeloid leukemia with induction failure. Leukemia. 2017;31(6):1306-1313.

11. Gounder MM, Zer A, Tap WD, et al. Phase IB study of selinexor, a first-in-class inhibitor of nuclear export, in patients with advanced refractory bone or soft tissue sarcoma. J Clin Oncol. 2016;34(26):3166-3174.

12. Machlus KR, Wu SK, Vijey P, et al. Selinexor-induced thrombocytopenia results from inhibition of thrombopoietin signaling in early megakaryopoiesis. Blood. 2017;130(9):1132-1143.

13. Etchin J, Berezovskaya A, Conway AS, et al. KPT-8602, a secondgeneration inhibitor of XPO1-mediated nuclear export, is well tolerated and highly active against AML blasts and leukemia-initiating cells. Leukemia. 2017;31(1):143-150. 